\title{
Research on the Practice of Commercial Illustration in the Park Culture under Win - Win Mechanism
}

\author{
Hu Maosheng \\ Chongqing Business Vocational College, Chongqing, China \\ hmshumaosheng@163.com
}

Keywords: Practice, Commercial Illustration, Park Culture, Win - win Mechanism

\begin{abstract}
In China, at the early stages of reform and opening up and towards a market economy, commercial illustrations resurrected in China. At the same time, in the long-awaited cultural and creative industry trends promote China in 2006 decided to take the pace of cultural and creative industries to promote Chinese manufacturing industry to upgrade and curb the environment to improve pollution, which opened the independent innovation the development of cultural and creative prelude.
\end{abstract}

\section{Introduction}

Culture is one of the biggest concerns of the Chinese government outside the economic issue. Since the reform and opening up three decades, a large number of international quality brands into China, foreign different cultures also entered the Chinese market. Especially in the field of animation and popular products, the United States, Japan, Europe dominated the Chinese market, China appeared 'cultural loss' and 'culture into the super phenomenon. The domestic market is not only for the occupation of foreign brand products, Chinese traditional culture is also gradually eroded by the market. In this issue, the illustrator is an animation and popular products, a direct image of the performance techniques, can effectively show their own characteristics of culture. Therefore, the development of Chinese illustrations industry can help to counter and offset this cultural product market gap effect. In addition to the animation industry and the digital game industry, in the design, advertising, and publishing industry, illustrations are closely related to a work, whether in the creative process or the final manufactured goods, illustrations are a common element, so vigorously develop Chinese illustrator industry, the overall cultural and creative industries will play a role in promoting.

\section{Basic Overview of Illustration Design}

Social development and commercial activities are now developing rapidly, the rapid development momentum, the activities of the commercial nature of the activities of the illustrations produced, so many people for commercial illustrations made a general definition, all forms of business activities for the commercial illustrations are called commercial illustrations, commercial illustrations are not entirely contained in the art of the range, it includes most of the work are emotional art, but the business illustrations are only for the business services, commercial illustrations have very strong purpose, and strong binding. Through the above description of commercial illustrations, the concept of ideas for commercial illustrations is defined as: commercial illustrations, a means of using intuitive vision in business activities to achieve the purpose of communicating valid information, the shape of the image, it is to achieve the content of its commitment to people with a very simple, clear image of the visual convey the purpose of a form. Commercial illustrations are divided into the following two categories: the first is the value of entertainment to achieve the commercial value of illustrations. For example: cartoon images, illustrations, cartoons and so on. It is mostly used in network forms, web applications, online games, 
paintings, movies or TV cartoons and derivative goods such as toys, animated branded merchandise. This kind of commercial illustration of the creation is not necessarily able to directly achieve its commercial value, generally there is no direct service object, its commercial value is to rely on professional post-marketing operations to achieve. The second is to realize the commercial value of the illustrations of artistic landscaping. They are in a variety of forms, such as books, magazines, newspapers, illustrations or product packaging and corporate images, product advertising, web production, television and movies. Compared with the former, the above-mentioned commercial illustrations are generally clear to the customer service and the purpose of creation, commercial illustrations of the contents of the general services directly to the organization of the enterprise.

\section{The Creative Illustration of Commercial Illustrations}

Most of the commercial illustrations used in the performance of the use of objective things is the use of this feature, determines the expression of commercial illustrations must pay attention to practical and popular. The key to the success of commercial illustrations is the importance of designing ideas and communicating with customers, creative media and materials because it can help viewers understand and appreciate illustrations correctly. In most cases, the perfect idea and the appropriate media through the visual form of expression can get the best results. It can be seen, therefore, that the creation of media and creativity in commercial illustration design has the same important position. Many illustrators have their own preferences, they will be based on their own needs to choose their own materials, their choice of materials, tools and technical differences so that their works have their own style. Continuous attempt is also the work of the new break code, but also to make the work of the unique way. However, the performance of commercial illustrations are mainly divided into realistic way, abstract and mixed three forms. Handwriting realistic way: the same as the traditional artistic approach, mainly to hand-based, can make sketches, oil paintings, ink, although in the digital age, the benefits of computer technology as a creative tool has now been widely recognized, but if the lack of tradition media, illustrators as nothing traditional media also have a computer cannot achieve the human nature, the original psychological feeling. The popularity of digital media still cannot replace the traditional hand-painted realistic way and it can only be called hand-painted illustrations of the "digital" performance. So, even if the digital media to commercial illustrations bring a broader space for development, but in the commercial illustrations of the market, the traditional realistic hand-painted illustrations of the status is still unshakable.

Collage style: pop art is a new art form prevalent in the international art scene from year to year and collage is the common technique of pop art, with different materials, cloth, paper, photos, feathers, toys and various a variety of waste combinations collage together. This fundamentally changed the illustrations of the creation of illustrations to the commercial illustrations of the impact is very far-reaching. Is an important representative of Pop Art, in his creation of "Marilyn Monroe" this work, in this piece of work, he is the unfortunate sexy Maema movie star Marilyn Monroe head for the creation of elements will be repeated her head, this painting in the color changes and tidy monotonous image, reflecting the social confusion and commercial helpless emptiness. As a result of the development of science and technology makes color copiers, digital cameras, scanners, computer and software development applications to give pop art new vitality.

Visual communication is the basic function of illustrations, the information conveyed mainly in the form of text bearing. Text is written language from writing language to visual language conversion is the essence of commercial illustrations. Illustrators must convert the written language into visual language according to the customer's text. These words may be just a few words of ad copy, it may be a novel content summarized into a few paintings. No matter what kind of text, and ultimately, should be the original text of the content of induction, hoof election, refining the material for the visual modeling. Language is an abstract concept, in the art of "means of expression" meaning. Text language is only the organization of text symbols and it is to convey logical thinking and image of the media. Among them, the image of literary language is not intuitive, with the help of the reader's perception and access. The illustrator uses the visual language and the 
illustrator will perceive the results of the association in an intuitive form to resort to the picture. Therefore, the text language and visual modeling language is the key to the success of commercial illustrations. General business illustrations creative process is divided into the following steps (to myself for everyone to create "everyone big brother" case process as an example): life: to receive all network customer needs, in the life before the creation, first of all Like the customer to confirm the nature of the work, the required style, the workload and as of the date. Communication: agree with the customer after the communication, to discuss, to determine the direction of customer requirements and ideas, communication and hope that the creation of the image of the big brother as follows: "There are problems to find everyone big brother, as the name of big brother name, as Everyone is a hot topic of the big masters, he is friends of the most ferocious friends! Although not an encyclopedia of academic theory, but he must be most concerned about the network hot spots most concerned about the hard work of the network, the latest hottest network topic, the most close to the life of the big friends, big brothers first time clairvoyant attention. We build everyone big brother will: create a new, understand the academic, then homely, talk feelings, after all, when Students are doing white - collar youth.

\section{Thoughts on the Development of Specialization}

World illustrations are involved in the development of today's global cultural and creative areas, and their creative images provide an effective range of industries. China has carried out a wide variety of cultural and creative industries on a broad dimension, and visual images are certainly indispensable rings, and their demand for quality and quantity in illustrations is equally high. But engraved under the market can be seen in the development of the figure is visible, but less of its quality. Under a variety of factors form a 'pan professional' phenomenon led to the promotion of the level of illustrations difficult. The illustrations of the country to think about the professional development of the words, the development of the world's regional illustrations of the process, and the industry's professional marketing operations to make some research and comparison, demonstration and practice of its accumulated more than 100 years of professional development experience. It is necessary to understand what is the common use of the world, which is subject to the country's national conditions and changes in the part of the need for Chinese illustrators to use wisdom, a good resolution of what can be directly moved to the application, What is necessary to change the face of the country to the situation.

Whether the illustrations of the region are specialized, can be measured from several aspects, not just look good painting. The key points to be concerned include the level of work, the efficiency of the operation, the industry standard, the social organization, the economic benefits, the education and training, the cultural and artistic value and the social participation, as the development guideline. From the following chart can be made more clearly and detailed description of the Chinese illustrator for the establishment of professional mechanisms, the various elements within the chart should not be ignored. For the current situation, in the chart, the key items should be the level of work, operating mechanism, professional norms, community organizations and education and training, will be discussed in detail here. The first is the requirement of the level of the work, which is a matter of course.

In the relevant Chinese illustrators can refer to some of the international illustrations in the operation of the practice, but also includes the signing of the contract, work guidelines, receipts and other items. The latter two issues and skills are applicable to the Chinese market. This is the means by which the illustrator applies the law to protect the rights and interests of one's own work, which should be based on the difference between Chinese provisions between the two international copyright laws. Chinese illustrators should note that it is impossible to carry out illustrations in foreign countries without signing a work contract. It is impossible to submit a work without a contract, which is universal. Legal common sense, but people often have to face and face on the grounds that the contract is not tight, to the final loss of the illustrator is always their own, and few customers are deceived by the illustrator to deceive things happen. Today, at this stage there are a lot of illustrators out of the oppression of the incident, the joint eye is also the work of the signing 
of the contract signed. Party know the size of the business world of the sale of the appointment must be orders, small to the street engraved ten RMB twenty RMB work also need to invoice, illustrator work is no reason not to implement these conventional items. For the Chinese illustrations of the professional process which is the most basic part of a link.

\section{Conclusion}

The illustrations have always been the kind of graphic art expression that is good at making others' narratives, which has witnessed the historical process of human civilization, documented the evolution of human culture and the commercial task of promoting economic development. In the inheritance and development of human culture, is an effective carrier, in the improvement of people's livelihood, it produced economic benefits. Since ancient times, illustrations in human culture and life have produced an important value, and in today's human life, it can have a greater impact to play its cultural and economic development of the special features.

\section{References}

[1] Wang Ling.Application of Popular Images in Contemporary Chinese Pop Art [J]. Art and Design (Theory) 2010 (01)

[2] Chen Xi. On the application of commercial illustrations [J]. Fine art. 2009 (02)

[3] Chen Xiu. New Illustrations of Commercial Illustrations [J]. Popular Literature (Theory). 2008 (08)

[4] Jin RMBpu. Contemporary world creative industry concept and its characteristics [J]. Film Art. 2006 (03)

[5] Shu Yi. Digital commercial art illustrations of the development and status quo [J]. Decorations. $2005(01)$

[6] Chen Chen. Bart's semiotic study of media culture [J]. Journal of Urumqi Vocational University. $2002(04)$ 